\title{
Effects of feeding chevon modified by dietary supplementation of Andrographis paniculata in rats as bioindicators
}

\begin{abstract}
This study examined the effects of chevon-based pellets prepared from Longissimus thoracis muscle of Boer goats fed diet supplemented with Andrographis paniculata leaves (APL), Andrographis paniculata whole parts (APWP) and control (AP0) on serum lipid profile in rats. Sixty male Sprague-Dawley rats (4-5 months old) were randomly assigned to pellets containing either APWP chevon, APL chevon, AP0 chevon or rat chow powder and fed for 12 weeks. Serum lipid profile was assessed at 0,6 and 12 weeks. Chevon-based pellets contained higher monounsaturated fatty acids than the rat chow powder. Plasma total cholesterol was higher $(\mathrm{P}<0.05)$ in rats fed AP0 chevon compared with those fed other diets at week 6. At weeks6 and 12, the APWP and APL rats had lower $(\mathrm{P}<0.05)$ total plasma cholesterol compared with AP0 and chow powder rats. At week 6, the level of HLDcholesterol in rats fed APWP chevon was higher $(\mathrm{P}<0.05)$ than those fed other dietary treatments. At week 12, the APL and APWP rats had higher $(\mathrm{P}<0.05)$ plasma HDLcholesterol than the chow powder and AP0 rats. At week 12, plasma LDL-cholesterol in rats fed AP0 meat was higher $(\mathrm{P}<0.05)$ than those fed other dietary treatments. At week 6 , the APL and APWP rats had lower $(\mathrm{P}<0.05)$ triglycerides than the chow powder and AP0 rats. At week 12, the plasma triglycerides in rats fed chow powder was higher $(\mathrm{P}<0.05)$ than those of rats fed other dietary treatments. Dietary APL and APWP chevon can modulate serum lipid profile in rats.
\end{abstract}

Keyword: Andrographis paniculata; Chevon; Serum; Cholesterol; Fatty acid; Rats 